Ann. Biol. anim., Bioch., Biophys., I966, 6 (2), I79-I88.

\title{
EFFETS COMPARÉS D'UN RÉGIME PROTÉIPRIVE ET D'UN RÉGIME CONTENANT UNE PETITE QUANTITÉ DE PROTÉINES D'GUF SUR L'EXCRÉTION D'AZOTE CHEZ LE RAT
}

\author{
Denise HUGOT et J. CAUSERET \\ Laboratoire d'Étude des Qualités biologiques des Aliments de l'Homme, \\ Centre national de Recherches zootechniques, 78 -Jouy-en-Josas
}

\section{SOMMAIRE}

Les effets respectifs d'un régime protéiprive et d'un régime à 4 p. roo de protéines d'œuf sur l'excrétion fécale et urinaire d'azote ont été étudiés chez le Rat adulte et chez le Rat en croissance.

I $^{0}$ Chez le Rat adulte, l'inanition azotée abaisse légèrement le niveau de l'ingestion énergétique. Chez le Rat en croissance, l'abaissement est beaucoup plus marqué.

$2^{\circ}$ L'excrétion d'azote fécal, chez le Rat adulte et chez le Rat en croissance, est plus élevée avec le régime contenant des protéines d'œuf qu'avec le régime protéiprive. Cet effet ne paraît pas dû aux différences observées dans le niveau de l'ingestion énergétique : car l'alignement de la consommation de nourriture des sujets soumis au régime contenant des protéines d'œuf sur celle des sujets en inanition azotée laisse subsister un surplus d'azote dans les fèces des premiers. II semble donc que l'utilisation digestive des protéines d'œuf soit incomplète.

$3^{\circ}$ L'excrétion d'azote urinaire, chez le Rat adulte, est sensiblement la même avec les deux régimes. Mais chez le Rat en croissance, elle est moins élevée avec le régime contenant des protéines d'œuf. Cet effet semble s'expliquer par les différences observées dans le niveau de l'ingestion énergétique, car l'alignement de la consommation de nourriture des sujets recevant des protéines d'œuf sur celle des sujets en inanition azotée fait disparaître toute différence entre les excrétions d'azote urinaire obtenues.

Contrairement à d'autres auteurs, nous n'avons donc observé aucun effet d'épargne direct des protéines d'œuf vis-à-vis des protéines corporelles.

\section{INTRODUCTION}

L'évaluation de l'utilisation digestive " nette " des protéines et de leur valeur biologique par les méthodes biologiques implique la détermination de l'excrétion d'azote métabolique fécal et d'azote endogène urinaire. Certains auteurs effectuent 
cette détermination en soumettant les animaux à un régime protéiprive, tanđis que d'autres utilisent un régime contenant une petite quantité de protéines d'œuf (en général : de 3 à $4 \mathrm{p}$. Ioo).

Les effets respectifs des deux types de régimes sur l'excrétion d'azote ont fait l'objet d'observations nombreuses, dont l'interprétation n'est pas unanime.

Io Les discussions les plus intéressantes concernent l'évaluation de l'excrétion urinaire d'azote.

Dans certaines conditions d'expérience, cette excrétion est plus faible avec un régime contenant une petite quantité de protéines d'œuf qu'avec un régime protéiprive. WILLMAN et al. (I945), Stevenson et al. (I946), BRush et al. (I947) ont observé ce phénomène chez des rats adultes préalablement privés d'une grande partie de leurs réserves protéiques labiles par une période d'alimentation sans azote (I I jours), puis maintenus en inanition protéique ou soumis à un régime à 3,5-4 p. Ioo de protéines d'œuf. Enn revanche, ils n'ont obtenu aucun effet du même genre, en remplaçant les protéines d'œuf par des protéines de muscle (de Porc ou de Rat), de la caséine ou de la gélatine. L'effet spécifique des protéines d'œuf pourrait résulter de leur richesse en acides aminés soufrés, car la $d l$-méthionine exerce, sur l'excrétion urinaire d'azote, une action dépressive plus marquée que celle des autres acides aminés essentiels (BRUsh et al., I947).

De même, MILLER (I944), puis AlLISON et al. (I946, I947), travaillant sur des chiens en état de déplétion azotée, ont signalé que l'excrétion d'azote urinaire était moins élevée quand on administrait des acides aminés soufrés $(l$-cystine, $d l$-méthionine) ou une petite quantité de blanc d'œuf, qu'avec un régime protéiprive.

Toutes ces données peuvent faire penser que les protéines d'œuf sont mieux utilisées que les produits du catabolisme azoté qui prennent naissance au cours de l'inanition protéique (Swanson, cité par Mrtcher, , I94 $^{8}$ ). Cependant Mitchel, (I948, I962) doute que l'administration de protéines d'œuf ou d'acides aminés soufrés abaisse réellement l'excrétion d'azote endogène urinaire. Il souligne que les études comparatives citées plus haut ont porté sur des animaux soumis pendant une longue période préliminaire à un régime sans azote, mais riche en lipides ( $20 \mathrm{p}$. Ioo dans les expériences sur le Rat, 22 p. Ioo dans les expériences sur le Chien). Des conditions d'alimentation aussi particulières altèrent la fonction hépatique ( $\mathrm{L}_{1}$ et FREEMAN, I946), cette altération pouvant accélérer le catabolisme protéique. Dès lors, il y a lieu de se demander si l'effet dépressif de protéines riches en méthionine ou de la méthionine elle-même sur l'excrétion d'azote urinaire ne serait pas la conséquence d'un effet bénéfique sur la fonction hépatique.

Pour appuyer son argumentation, MrTchel, (I948, I962) rappelle qu'au cours de la plupart des études réalisées dans son laboratoire sur des rats adultes ou en croissance soumis à un régime protéiprive renfermant au maximum $12 \mathrm{p}$. Ioo de lipides, aucun abaissement de l'excrétion d'azote endogène urinaire n'a été obtenu par administration d'acides aminés isolés, de mélanges d'acides aminés ou de protéines d'œuf (MrTchel, et CARMaN, I926; TrEIChLER, I939; Burroughs et al., I940; BRICKER et MITCHELL, I947).

$2^{\circ}$ Les effets respectifs d'un régime protéiprive et d'un régime renfermant une petite quantité de protéines d'œuf sur l'excrétion fécale d'azote n'ont pas suscité de discussions analogues. 
I,e seul problème qui se pose actuellement est de savoir si l'incorporation de protéines d'œuf dans un régime protéiprive reste sans effet sur cette excrétion, ou si elle l'augmente. En se basant sur les résultats obtenus dans son laboratoire, MrTCHELL (I948) admet que, chez le Rat, les protéines d'œuf sont complètement ou presque complètement digestibles, en sorte que leur ingestion en petite quantité ne modifie pas sensiblement 1'excrétion d'azote métabolique fécal. MiTchELL, et BEADLES (I950) l'ont confirmé en expérimentant avec une préparation de poudre d'œuf dégraissée obtenue au laboratoire; mais, avec une préparation commerciale, ils ont observé une excrétion d'azote fécal plus élevée chez les sujets dont le régime contenait cette préparation au taux de $3 \mathrm{p}$. Ioo, que chez des témoins en inanition azotée.

Les observations personnelles rapportées dans le présent mémoire ont été effectuées sur des rats adultes ou en croissance initialement en bon état de nutrition, soumis soit à un régime protéiprive, soit à un régime contenant des protéines d'œuf, le taux de lipides atteignant I2 p. roo dans les deux cas. Ces observations portent à la fois sur l'excrétion d'azote fécal et sur l'excrétion d'azote urinaire.

\section{EXPÉRIENCE SUR RATS ADULTES}

\section{Méthode}

L'expérience porte sur cinquante rats Wistar mâles provenant de l'élevage du Laboratoire, nourris depuis le sevrage avec une provende commerciale qui contient $22 \mathrm{p}$. Ioo de protéines. Ils pèsent initialement de 250 à $330 \mathrm{~g}$, avec un poids moyen de $280 \mathrm{~g}$. On les répartit en deux groupes.

Les sujets du groupe I reçoivent, pendant 15 jours, un régime protéiprive, mais par ailleurs équilibré, présentant la composition centésimale suivante :

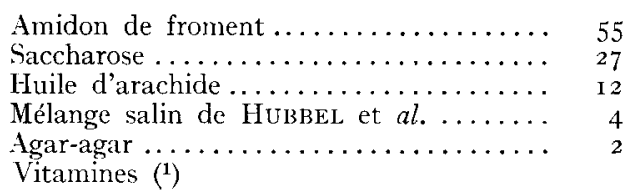

Ce régime contient $40 \mathrm{mg}$ d'azote pour ıoo g. Il est distribué ad libium, sous forme de bouillie obtenue en faisant chauffer 2 parties du mélange précédent avec I partie d'eau distillée, les vitamines n'étant introduites qu'après le chauffage. Les animaux reçoivent de l'eau distillée comme boisson.

Simultanément, les rats du groupe II sont soumis à un régime à 4 p. roo de protémes d'œuf, dérivant du précédent par introduction de 8 p. roo de poudre d'œuf entier ${ }^{2}$ ), par diminution du taux de l'huile d'arachide (9 p. I00 au lieu de I 2 p. I0o) en vue d'égaliser la teneur en lipides des deux régimes, et par ajustement du taux d'amidon de manière à atteindre le total Ioo.

Les fèces et les urines de chaque sujet sont recueillis durant deux périodes successives d'une semaine (du $2^{\mathrm{e}}$ au $8^{\mathrm{e}}$ jour, du $9^{\mathrm{e}}$ au ${ }_{1} 5^{\mathrm{e}}$ jour) $\left({ }^{3}\right)$, au moyen de dispositifs décrits antérieurement (CaUSeret, 1954). L'azote y est dosé, par microkjeldahl.

(1) Par $\mathrm{kg}$ de régime : thiamine $2 \mathrm{mg}$, riboflavine $3 \mathrm{mg}$, pyridoxine $25 \mathrm{mg}$, acide nicotinique $50 \mathrm{mg}$, pantothénate de calcium $10 \mathrm{mg}$, vitamine $\mathrm{K}$ hydrosoluble $\mathrm{I} \mathrm{mg}$. L'huile d'arachide du régime est additionnée de 1 p. I 00 d'huile de foie de morue titrant 2000 UI de vitamine A et 500 UI de vitamine D par ml, et de 0,015 p. roo d' $\alpha$-tocophérol.

${ }^{(2)}$ Euf complet Gallia en poudre spray. Pour le Rat en croissance, ce produit a une efficacité protidique égale à celle de l'œuf entier frais.

$\left({ }^{3}\right)$ Pour évaluer la perte d'azote endogène urinaire, Mitchell (I948) soumet les animaux à un régime contenant 4 p. roo de protéines d'œuf durant 2 semaines consécutives; l'excrétion urinaire d'azote est évaluée pendant la deuxième semaine. 


\section{Résultats}

Les résultats sont consignés dans le tableau $I$.

On voit que, dans les conditions d'expérience adoptées, 1'introduction de $4 \mathrm{p}$. Ioo de protéines d'œuf dans le régime protéiprive accroît de 22 p. Ioo l'excrétion d'azote fécal. Cet accroissement est dû vraisemblablement, au moins pour une part, à l'augmentation de la quantité de nourriture ingérée, augmentation qui est de 17 p. IoO. Il est possible qu'il s'explique aussi par la présence, dans les fèces des sujets qui reçoivent des protéines d'œuf, d'un surplus d'azote alimentaire non absorbé.

Quant à l'excrétion d'azote urinaire, sa valeur absolue n'est pas modifiée significativement par l'introduction de protéines d'œuf dans le régime. C'est seulement lorsqu'on la rapporte à la " taille métabolique " (puissance 0,75 du poids corporel) qu'elle apparaît un peu moins élevée, durant la $2^{\mathrm{e}}$ période, chez les sujets recevant des protéines d'œuf que chez les témoins.

\section{EXPÉRIENCES SUR RATS EN CROISSANCE,}

\section{IO EXPÉRIENCE PRINCIPAIE}

\section{Méthode}

Cent cinq rats Wistar mâles, provenant de l'élevage du Laboratoire, âgés initialement de 30 jours et pesant de 70 à $90 \mathrm{~g}$, sont répartis en trois groupes. Ceux-ci reçoivent respectivement :

(I) le régime protéiprive, ad libitum;

(II) le régime à $4 \mathrm{p}$. Ioo de protéines d'œuf, ad libitum;

(III) le régime à 4 p. Ioo de protéines d'œuf, en quantité restreinte alignée chaque jour sur la quantité moyenne que les sujets soumis au régime protéiprive avaient consommée spontanément la veille.

Ces régimes sont identiques à ceux de l'expérience sur rats adultes.

Le groupe (III) est institué pour tenir compte du fait que le Rat jeune réduit son niveau d'ingestion calorique beaucoup plus fortement que le Rat adulte, lorsqu'il est privé d'azote : l'anorexie s'accentue d'ailleurs avec le temps et entraîne une perte de poids de plus en plus marquée, avec issue mortelle en une quinzaine de jours (CAUSERET et al., 1965). Aussi la présente expérience ne dure-t-elle que 8 jours, le bilan azoté étant déterminé du $2^{\mathrm{e}}$ au $8^{\mathrm{e}}$ jour.

\section{Résultats}

D'après le tableau 2, l'introduction de protéines d'œuf dans le régime des ani maux alimentés ad libitum (groupe II) entraîne un fort accroissement de l'excrétion d'azote fécal. Cet accroissement est certainement dû pour une part à l'augmentation de la quantité de nourriture ingérée, augmentation qui atteint près de 60 p. Ioo. Mais il résulte vraisemblablement aussi d'une utilisation digestive incomplète des protéines d'œuf, puisque l'excrétion d'azote fécal est significativement plus élevée dans le groupe III que dans le groupe I, alors que le niveau d'ingestion calorique est le même dans les deux groupes.

Quant à 1'excrétion d'azote urinaire (exprimée en valeur absolue ou rapportée à la " taille métabolique »), elle est abaissée de 30 p. Ioo environ chez les animaux du groupe II consommant ad libitum le régime à 4 p. Ioo de protéines d'œuf. Mais l'alignement du niveau d'ingestion du même régime (groupe III) sur celui du régime 

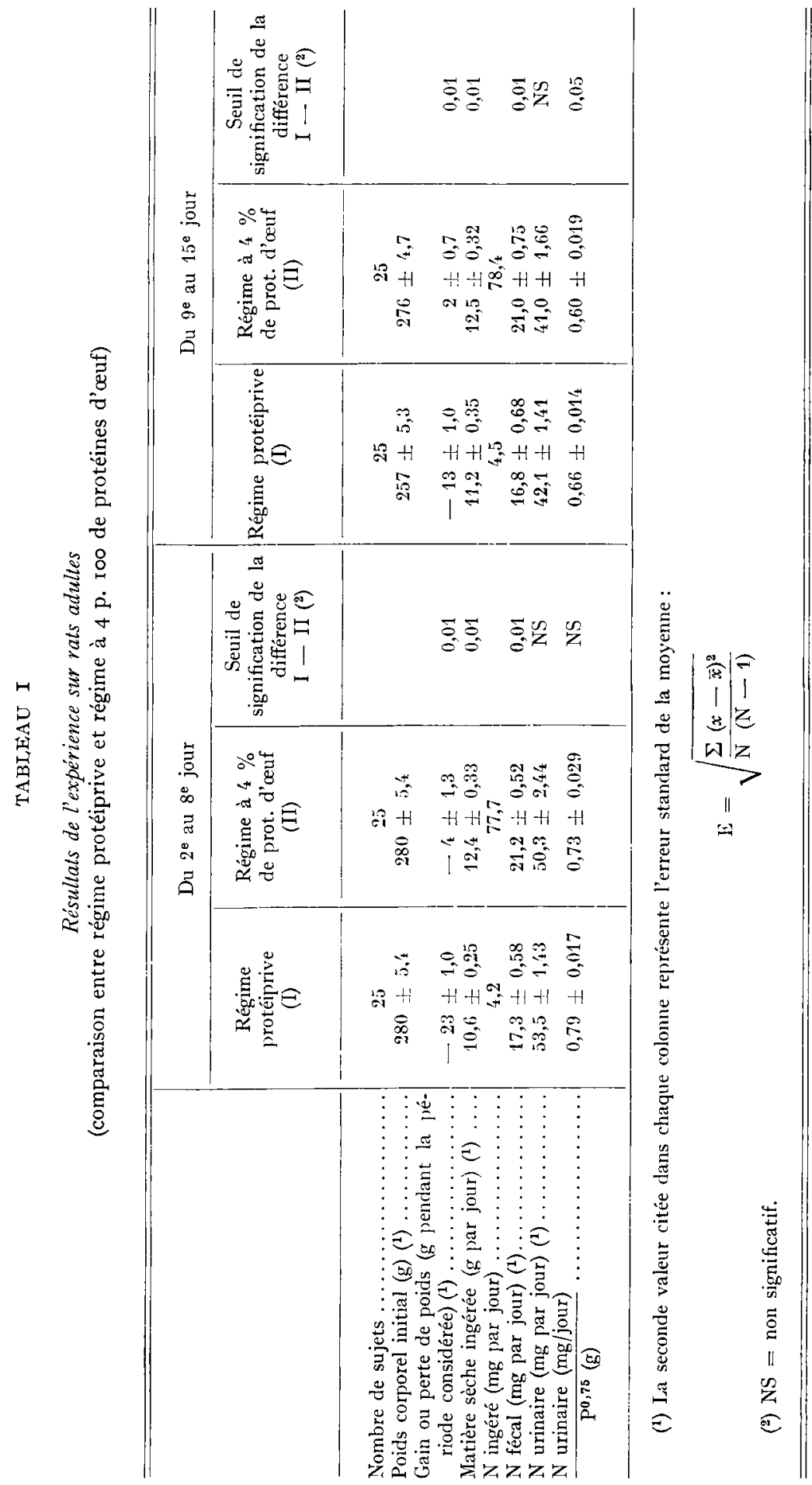
protéiprive (groupe I) fait disparaître toute différence entre les excrétions d'azote urinaire des rats qui reçoivent ces deux régimes.

On peut en déduire que la diminution de l'excrétion d'azote urinaire provoquée par les protéines d'œuf est due principalement au relèvement du niveau de l'ingestion calorique.

\section{TABLEAU 2}

Résullats de l'expérience principale sur rats en croissance

\begin{tabular}{|c|c|c|c|c|c|c|}
\hline & \multicolumn{6}{|c|}{$\mathrm{Du} 2^{\mathrm{e}}$ au $8^{\mathrm{e}}$ jour } \\
\hline & \multirow{2}{*}{$\begin{array}{l}\text { Régine } \\
\text { protéiprive } \\
\text { (1) }\end{array}$} & \multirow{2}{*}{\begin{tabular}{|} 
Régime à 's \% \\
de prot. d'ouf \\
ad libitum \\
(II)
\end{tabular}} & \multirow{2}{*}{$\begin{array}{l}\text { Régime à } 4 \text { c } \% \\
\text { de prot. d'ceuf } \\
\text { restreint }\left({ }^{2}\right) \\
\text { (III) }\end{array}$} & \multicolumn{3}{|c|}{$\begin{array}{l}\text { Seuil de signification } \\
\text { des différences }\left({ }^{3}\right)\end{array}$} \\
\hline & & & & $\mathrm{I}-\mathrm{II}$ & $I-I I I$ & II - III \\
\hline Nombre de sujets............. & 42 & 31 & 32 & & & \\
\hline Poids corporel initial $(\mathrm{g})\left({ }^{1}\right) \ldots$ & $92 \pm 1,7$ & $92 \pm 2,1$ & $93 \pm 1,6$ & & & \\
\hline Gain ou perte de poids (g pendant & & & & & & \\
\hline l'expérience) $\left({ }^{1}\right) \ldots \ldots \ldots \ldots \ldots$ & $-19 \pm 0,7$ & 0 & $-10 \pm 0,8$ & 0,01 & 0,01 & 0,01 \\
\hline Matière sèche ingérée (g par jour) (1) & $6,51 \pm 0,12$ & $10,3^{\prime} \div 0,22$ & $6,42 \pm 0,0$ & 0,01 & $\mathrm{NS}$ & 0,01 \\
\hline $\mathrm{N}$ ingéré (mg par jour)......... & 2,7 & 62, , $^{\prime}$ & 38,7 & & & \\
\hline $\mathrm{N}$ fécal (mg par jour) $\left.{ }^{1}\right) \ldots$ & $8,59 \pm 0,26$ & 11,32 上 0,35 & $10,83 \pm 0,36$ & 0,01 & 0,01 & 0,01 \\
\hline N urinaire (mg par jour) $\left.{ }^{(}\right)$ & $32,68 \pm 0,63$ & $23,31 \pm 0,92$ & $31,55 \pm 0,59$ & 0,01 & $\mathrm{NS}$ & 0,01 \\
\hline $\mathrm{N}$ urinaire (mg/jour) & $1,11 \div 0,02 / \mathbf{t}$ & $0,79+0,03_{t}^{\prime}$ & $1,05+0,016$ & 0,01 & NS & 0,01 \\
\hline $\mathrm{P}^{0,75}(\mathrm{~g})$ & $1,11=0,02 \mathrm{t}$ & $0, \lambda=0,0 \cdot 3$ & $1,00 \pm 0,010$ & 0,01 & $1 \times 5$ & 0,01 \\
\hline
\end{tabular}

(1) La seconde valeur citée dans chaque colonne rejrésente l'erreur standard de la moyenne.

$$
E=\sqrt{\frac{\sum(x-\bar{x})^{2}}{N(N-1)}}
$$

(2) Consommation alignée sur la consommation moyenne du groupe I.

( $\left.{ }^{3}\right)$ NS $=$ non significatif.

Toutefois, les sujets du groupe III ont ingéré une quantité de protéines d'œuf moins élevée que ceux du groupe II. On peut se demander si ce dernier fait ne porte pas lui aussi une part de responsabilité dans le relèvement de l'excrétion d'azote urinaire du groupe III par rapport à celle du groupe II. Une expérience complémentaire a donc été instituée en vue de répondre à cette question.

\section{$2^{O}$ FXXXÉRIENCE COMPLÉMENTAIRE}

\section{Méthode}

Quarante-huit rats Wistar mâles, provenant de l'élevage du Laboratoire, âgés initialement de 30 jours et pesant de 70 à $90 \mathrm{~g}$, sont répartis en quatre groupes :

- groupes I, II, III identiques à ceux de l'expérience principale sur rats en croissance ;

- groupe IV, à consommation de nourriture alignée sur celle des groupes I et III, mais recevant autant de protéines d'œuf que le groupe II. Pour obtenir ce résultat, la quantité de protéines d'œuf incorporée chaque jour dans la ration distribuée aux rats du groupe IV est alignée sur la quantité consommée durant la journée précédente par les sujets du groupe III.

Le bilan azoté est déterminé du $2^{\mathrm{e}}$ au $8^{\mathrm{e}}$ jour. 
PROTÉINES D'GUE E'T EXCRÉTION D'AZOTE
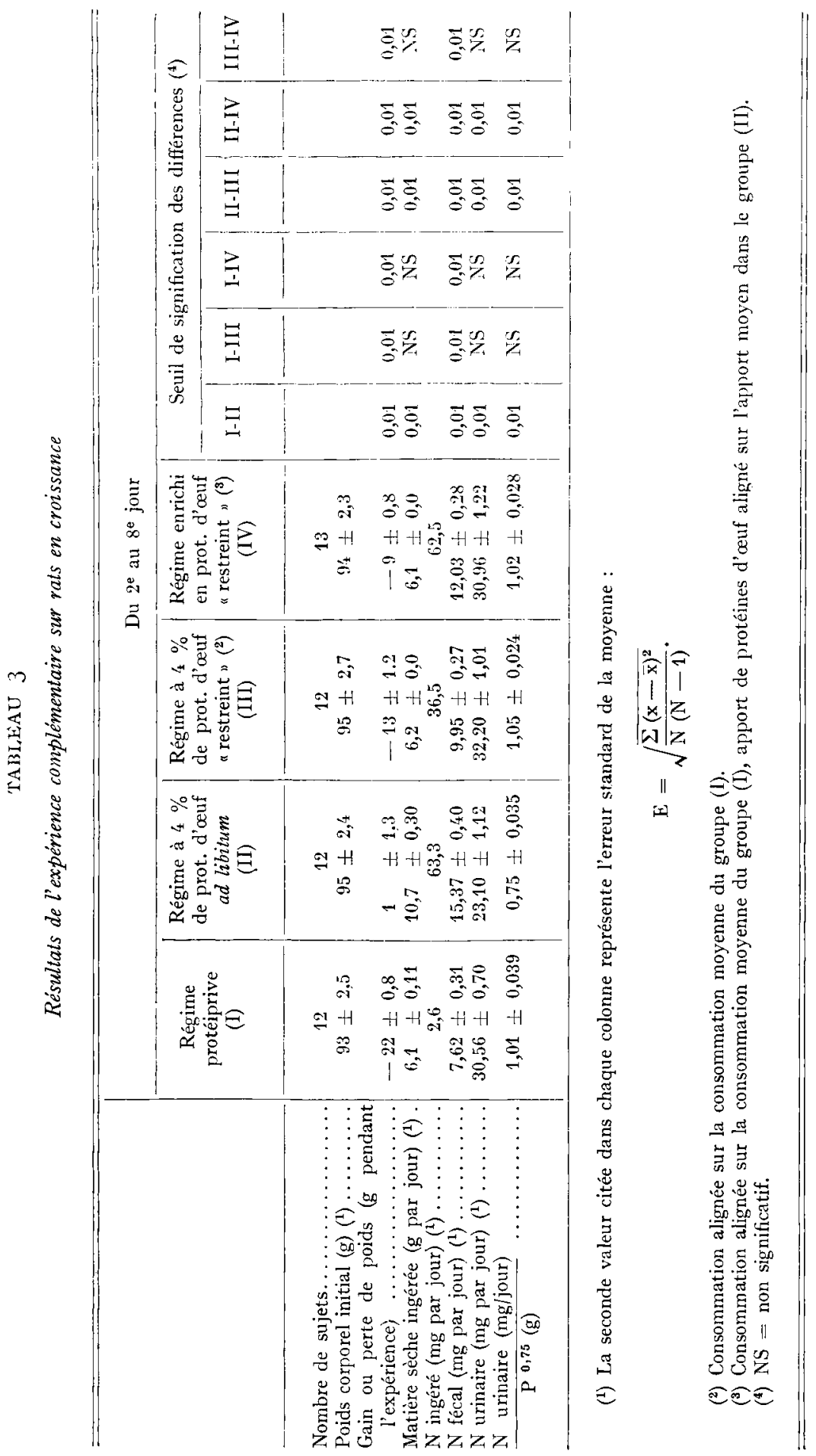


\section{Résultats}

D'après le tableau III, les résultats de l'expérience principale sont entièrement confirmés.

D'autre part, on constate que l'excrétion urinaire des sujets du groupe IV ne diffère pas significativement de celle des sujets du groupe I, mais qu'elle est supérieure à celle des rats groupe II (différence significative au seuil de $\mathrm{P}=0, \mathrm{O}$ ).

Dans les conditions d'expérience adoptées, on n'observe donc aucun effet d'épargne direct des protéines d'œuf vis-à-vis des protéines corporelles : sur ce point, nos conclusions coïncident avec celles de Míchels (I948, I962).

\section{DISCUSSION}

Io Comme on l'a fait remarquer, les résultats obtenus chez le Rat en croissance donnent à penser que l'utilisation digestive des protéines d'œuf est incomplète.

On pourrait objecter à cette conclusion, que le surplus d'azote obtenu chez les sujets soumis au régime contenant des protéines d'œuf (par rapport'à l'excrétion azotée des animaux qui consomment le régime protéiprive en quantité égale) provient peut-être des sucs digestifs, et non du régime. Toutefois, il est généralement admis que la stimulation des sécrétions digestives est sans effet sur l'excrétion d'azote métabolique fécal (MITcheiL, I948).

Calculé d'après nos résultats, le coefficient d'utilisation digestive des protéines d'œuf atteint :

- chez le Rat adulte : 94,7 pendant la première semaine d'expérience et 94,3 pendant la seconde ;

- chez le Rat en croissance : 90,4 ou 93,6 suivant qu'on base le calcul sur les résultats des groupes I et II ou des groupes I et III (le dernier mode de calcul paraissant préférable).

Ces valeurs correspondent à celle que nous avons obtenue, par ailleurs, chez le jeune Rat soumis successivement à un régime protéiprive et à un régime à Io p. Ioo de protéines d'œuf : soit 93,0 (résultats non publiés). Elles concordent aussi avec les valeurs mentionnées par d'autres auteurs : 9I,5 ou 95,4 chez la jeune Souris suivant que le régime contenant des protéines d'œuf est consommé ad libitum ou que sa consommation est alignée sur celle du régime protéiprive (BOSSHARDT et BARNES, I946) ; 95,4 chez le jeune Rat pour les protéines du vitellus (LAPORTA, I939).

En conséquence, s'il peut exister des préparations à base d'œuf entier dont les protéines ont un coefficient d'utilisation digestive réel de Ioo p. Ioo, il ne parait pas possible d'attribuer cette propriété aux protéines d'œuf en général.

$2^{0}$ En ce qui concerne les effets respectifs du régime protéiprive et du régime à $4 \mathrm{p}$. Ioo de protéines d'œuf sur l'excrétion d'azote urinaire, il ne semble pas qu'il $\mathrm{y}$ ait d'opposition entre les résultats obtenus chez les animaux adultes et chez les jeunes.

Il est probable que, chez les adultes soumis au régime protéiprive, la faiblesse de la diminution de la consommation de nourriture et l'existence de réserves grasses permettent à l'organisme de couvrir son besoin d'énergie sans faire appel à ses protéines tissulaires : l'excrétion d'azote urinaire est donc à peine augmentée. 
Au contraire, chez les jeunes soumis au même régime, l'anorexie qui s'installe dès les premiers jours et la faiblesse des réserves grasses obligent vraisemblablement l'organisme à utiliser ses propres protéines corporelles comme sources d'énergie ${ }^{1}$ ): d'où l'augmentation de l'excrétion d'azote urinaire.

$3^{\circ}$ Nos observations conduisent à penser que, pour la détermination de l'utilisation digestive nette des protéines et pour celle de leur valeur biologique chez le Rat adulte, il n'y a pas lieu d'administrer un régime contenant une petite quantité de protéines d'œuf, plutôt qu'un régime protéiprive.

Chez le Rat en croissance, il semble en être de même pour l'estimation de l'excrétion d'azote métabolique fécal. En revanche, l'évaluation de l'excrétion d'azote urinaire est plus facile à réaliser et probablement plus exacte si l'on emploie un régime à 3 ou 4 p. Ioo de protéines d'œuf. Ce régime permet en effet d'éviter la sousalimentation, qui entraîne elle-même :

- l'obligation de ne soumettre les animaux qu'à une période d'adaptation très courte avant d'effectuer la mesure ;

- l'élimination d'un surplus d'azote par les reins;

- la nécessité, après la période d'alimentation sans azote, de soumettre les animaux à un régime complet et d'attendre qu'ils aient recouvré leur poids corporel initial pour leur administrer les protéines étudiées.

\section{Reçu pour publication en fórier 1966.}

\section{SUMMARY}

THE COMPARATIVE EFFECTS OF A PROTEIN-FREE DIET AND A DIET CONTAINING A SMALL AMOUNT OF EGG PROTEINS ON EXCRETION OF NITROGEN BY TIIE RAT

The effects of a protein-free diet and of one with 4 per cent egg proteins on excretion of nitrogen in fæces and urine were studied with adult and growing rats.

I. In adult rats deprivation of protein slightly reduced intake of energy. In growing rats the reduction was much greater.

2. In both adult and growing rats more nitrogen was excreted in fæces on the diet with egg proteins than on the protein-free diet. This effect did not seem to be due to the observed difference in intake of energy, since in the young rat when intakes of the protein-free diet and of the diet with egg proteins were equalized there remained a greater amount of nitrogen in fæces of the rats given the diet with egg proteins. It seemed then, that digestive utilization of egg proteins was not complete.

3. In the adult rat the excretion of nitrogen in urine was very similar on both diets. In the growing rat it was less on the diet with egg proteins. This effect seemed to be explained by the differences in intake of energy, since when intake of feed was equalized for the rats on the diet with egg proteins and those deprived of nitrogen the difference in excretion of nitrogen in urine disappeared.

In contrast to other authors, we have found no direct sparing effect of egg proteins on body proteins.

\section{RÉFÉRENCES BIBLIOGRAPHIQUES}

Allison J.B., Seeley R. D., Brown J. H., ANderson J. A., 1946. The evaluation of proteins in hypoproteinemic dogs. J. Nutr., 31, $237-247$.

Allison J. B., Anderson J. A., Seelky R. D., 1947. Some effects of methionine on the utilization of nitrogen in the adult dog. J. Nutr., 33, $361-370$.

(1) En deux semaines, les animaux perdent environ 25 p. 100 de leur poids initial. 
I3osshardt D. K., BARNes R. H., I946. The determination of metabolic fecal nitrogen and protein digestibility. J. Nulr., 31, I3-21.

Bricker M. L., Mitchell H. Il., 1947. The protein requirements of the adult Rat in terms of the protein contained in egg, milk and soy flour, J. Nutr.. 34, 49I-505.

Brush M., Willman W., Swhrson P. l'., 1947. Amino acids in nitrogen metabolism with particular reference to the role of methionine. $J$. Nulr., 33, 389-410.

Burroughs E. W., Burrougiss H. S., Mitchell H. H., r940. The independence of the endogenous and the exogenous metabolism of nitrogen. J. Nutr., 19, 27 I-283.

Causeret J., 1954. Recherches sur l'utilisation physiologique du calcium au cours de la croissance chez le Rat. Ann. Zootech., 3, 27I-336.

Causeret J., Hugot D., Arnoux J., 1965. Étude statistique des variations de l'excrétion d'azote métabolique fécal et d'azote endogène urinaire en fonction du poids corporel, du poids des ingesta et du poids des féces chez le jeune Rat. I. En régime protéiprive. Ann. Biol. anim. Bioch. Biophys., 5, 6I-78.

Laporta M., BUX G., Piccoli R., i 938. Valore biologico dei protidi del tuorlo d'uovo. Quad. Natrisione, $5,3^{8-42}$.

I I T. W., Freeman S., I946. Effect of protein deficiency and cholesterol feeding on the liver of dogs. Ames. J. Physiol., 145, 646-659.

Miller L. L., x944. The metabolism of $a l$-methionine and $l$-cystine in dogs on a very low protein diet. J. biol. Chem., 152, 603-61 1 .

Mitcield H. II., r948. The biological utilization of proteins and protein requirements. In SaHYCX M. Proteins and amino acids in nutrition, $46-8 \mathrm{I}$, Reinhold Publ. Co, New York.

Mitchell H. II, I962. Comparative nutrition of man and domestic animals, vol. I, I5I-152, Academic Press, New York.

Mitciell H. H., Beadles J. R., I950. Biological values of six partially purified proteins for the adult albino rat. J. Nutr., 40, $25-40$.

Mitchell H. H., Carman G. G., I926. The biological value of the nitrogen of mixtures of patent white flour and animal foods. J. biol. Chem., 68, I83-215.

Stevenson G. T., Swanson P. P., Wiltman W., Brusil M., ig46. Nitrogen metabolism as influenced by level of caloric intake, character of diet and nutrition state of animal. Fed. Proc., 5, $240-24 \mathrm{I}$.

TREICHLER R., I939. Effect of age and other factors on the relationship between basal metabolism and endogenous nitrogen melabolism. Ph. D. Thesis, Lniv. Illinois, $65 \mathrm{p}$.

Willman W., Swanson P. P., Stewart G. F., Stevenson G. T., Brush M., i945. Biological efficiency of egg proteins. Proc. Fed. Amer. Soc. Exper. Biol., 4, i64. 